\title{
Una propuesta para un sistema de análisis técnico para compra y venta de acciones en la Bolsa Mexicana de Valores
}

\author{
Alfredo Díaz Mata*
}

\section{Resumen}

Se presenta el procedimiento y el conjunto de herramientas del análisis técnico que utiliza un sistema computarizado que se está construyendo con el propósito de tomar decisiones de compra y venta de acciones en la Bolsa Mexicana de Valores (BMV), así como también los resultados alcanzados en 14 meses.

El sistema consiste, básicamente, en realizar simulaciones y evaluaciones sobre los precios de 29 acciones de alta bursatilidad de la Bolsa Mexicana de Valores (BMV) y sobre los valores del Índice de Precios y Cotizaciones (IPC) de la propia BMV y del Dow Jones Industrial Average (DJIA) de la Bolsa de Valores de Nueva York, utilizando herramientas del análisis técnico bursátil y con base en dos programas de cómputo; uno de ellos es el Metastock, un programa comercial de análisis técnico y el otro es uno que está siendo desarrollado por el autor, con ayuda de especialistas, específicamente en Visual Basic 6.0 para incluir los mecanismos propuestos en el sistema que se presenta aquí.

Palabras clave: análisis técnico, Bolsa Mexicana de Valores.

* Investigador de la División de Investigación de la Facultad de Contaduría y Administración, UNAM. Correo electrónico: alfredimata@hotmail.com 


\section{INTRODUCCIÓN}

耳 $\mathrm{n}$ este trabajo se propone, como se menciona en el resumen, un procedimiento

- computarizado para toma de decisiones de inversión en acciones de la Bolsa Mexicana de Valores, basado en mecanismos del análisis técnico. Esta propuesta es el punto en el que se encuentra, hacia finales de enero de 2004, una investigación que pretende, precisamente, construir un sistema de esa forma y que es uno de los objetivos primordiales de los estudios que estoy realizando para obtener el grado de Doctor en Administración en la Facultad de Contaduría y Administración (FCA) de la Universidad Nacional Autónoma de México (UNAM).

Los antecedentes directos de esta propuesta son primordialmente mis experiencias anteriores con el análisis bursátil en general y con el análisis técnico en particular y que se pueden resumir en dos de los libros que he escrito (Matemáticas Financieras e Invierta en la Bolsa), en los artículos que he presentado sobre el tema en la revista Contaduría y Administración y en la tesis que escribí, también sobre el mismo tema, para obtener el grado de Maestro en Finanzas, en la propia FCA de la UNAM. En la bibliografía del final están los detalles.

El sistema se basa en el análisis de las siguientes 29 acciones de alta bursatilidad ${ }^{1}$ de la BMV:

\section{Tabla 1}

\begin{tabular}{|ll|ll|l|} 
ALFAA & CELV & FEMSAUBD & GMEXICOB & TELMEXL \\
\hline AMXL & CEMEXCPO & GCARSOA1 & GMODELOC & TLEVISACPO \\
APASCO* & CIEB & GEOB & ICA* & TVAZTCACPO \\
\hline ARA* & COMERCIUBC & GFBBO & KIMBERA & WALMEXC \\
\hline BIMBOA & CONTAL & GFINBURO & SORIANAB & WALMEXV \\
\hline C & ELEKTRACPO & GFNORTEO & TELECOMA1 & \\
\hline
\end{tabular}

\footnotetext{
${ }^{1}$ Esta categoría de "alta bursatilidad" varía según las operaciones, por lo que vale la pena puntualizar aquí que las acciones incluidas en el estudio se encontraban en esa categoría según los reportes de acciones que aparecían en el periódico El Financiero hacia principios de 2002.
} 
Una propuesta para un sistema de análisis técnico para compra y venta de acciones en la Bolsa Mexicana de Valores

Además de las 29 acciones anteriores, se analizan también dos índices accionarios para evaluar el mercado en su conjunto. Estos índices son el Índice de Precios y Cotizaciones (IPC) de la BMV y el Promedio Industrial Dow Jones (DJIA) de la Bolsa de Valores de Nueva York (NYSE).

La parte fundamental de este sistema que se propone para tomar decisiones con el objeto de comprar y vender acciones es el procedimiento que se sigue para el análisis y que consta de una serie de actividades que se llevan a cabo todos los días hábiles, por lo general al cierre de cada día de operación, es decir, a partir de las 4 o 5 de la tarde, de lunes a viernes, exceptuando días festivos.

Las actividades que resumen el sistema, y que se explican con detalle más adelante, son:

1. Se actualiza la base de datos con los precios y volumen de cierre del día de las 29 acciones y los dos índices de mercado.

2. Se realizan simulaciones con el oscilador estocástico \% K para encontrar los valores óptimos de los parámetros y buscar si se generan señales de compra o venta. Estas simulaciones se llevan a cabo con el programa construido con Visual Basic, utilizando, por lo general, periodos de tres meses.

3. Se revisan, en gráficas construidas con el paquete Metastock, los indicadores direccionales ID+ e ID- para ver en qué posición se encuentran y, en consecuencia, si dan señales de compra y/o de venta.

4. Para las acciones que generan señales:

a) Se determina si hay una confirmación de las señales de los dos indicadores técnicos anteriores, $\% \mathrm{~K}$ e indicadores direccionales. En otras palabras, se revisa si alguna acción tiene señales de compra o de venta con ambos indicadores.

b) Se revisa si se presentan brechas en los precios.

c) Se revisa si se presentan días clave de reversión, de una o de dos barras.

5. Finalmente, se evalúa la tendencia del mercado, a través del IPC y del DJIA, utilizando los mismos indicadores mencionados en los apartados anteriores.

En la siguiente sección se detalla cada uno de estos pasos, a los que se ha denominado "elementos". 


\section{LOS ELEMENTOS DEL PROCEDIMIENTO}

Se revisan a continuación los principales componentes del sistema que se propone.

\section{La base de datos y su actualización}

Se utilizan sólo acciones de alta bursatilidad porque son las que mejor permiten lograr efectivamente las operaciones de compra y venta y, también, porque permiten alcanzar precios cercanos a los de cierre que se asumen en este análisis.

Se requiere la actualización diaria porque las decisiones de compra o venta se toman sobre la misma base: todos los días. La base de datos contiene precios de apertura, máximo, mínimo y cierre y, además, el volumen de operaciones para cada día hábil y para cada acción o índice. Estos datos incluyen, en su mayoría, fechas desde 1995 o anteriores.

Se tienen dos bases, una que se utiliza con el paquete Metastock y otra en Excel para su uso con un programa en Visual Basic 6.0 específicamente elaborado para realizar simulaciones para el sistema que aquí se propone y al que en lo sucesivo se denomina "simulador VB 6". Ambas se actualizan diariamente con datos que se "bajan" del sector de finanzas del sitio de Internet de Yahoo! y se obtienen en formato de Excel.

\section{Las simulaciones con el oscilador estocástico $\% \mathrm{~K}$}

El oscilador estocástico se calcula como:

$$
\% K_{i, n}=100\left(\frac{C_{i}-\operatorname{Min}_{n}}{\operatorname{Max}_{n}-\operatorname{Min}_{n}}\right)
$$

en donde,

$\mathrm{i}$ = es la sesión (día) para la que se calcula el oscilador

$\mathrm{n}=$ número de periodos (días también) utilizados para el cálculo

$C_{i}=$ precio de cierre de la sesión

$\operatorname{Max}_{n}=$ precio máximo de los $\mathrm{n}$ periodos

$\operatorname{Min}_{n}=$ precio mínimo de los $\mathrm{n}$ periodos 
Una propuesta para un sistema de análisis técnico para compra y venta de acciones en la Bolsa Mexicana de Valores

Como puede verse en la fórmula, se trata de un oscilador de tasa de cambio porque mide el cambio relativo (porcentual) de las diferencias entre a) último precio de cierre y precio mínimo del periodo, contra b) precios máximo y mínimo, o sea el rango total de variación de los precios del título. Y, a su vez, esto implica que el oscilador mide, primordialmente, el impulso o momentum ${ }^{2}$ de los precios. Su inclusión en el sistema se desprende de análisis previos, tal como se explica en los párrafos siguientes.

El propósito principal de la tesis que elaboré para obtener el grado de Maestro en Finanzas $^{3}$ fue abordar el análisis técnico aplicado al corto plazo en el mercado accionario mexicano, haciendo especial hincapié en la posibilidad de estructurar un sistema de decisiones de inversión totalmente computarizado, utilizando el paquete Metastock, que prescindiera de la parte del análisis gráfico que se utiliza tradicionalmente en el análisis técnico y que permitiera superar por un lado el comportamiento del Índice de Precios y Cotizaciones (IPC) de la Bolsa Mexicana de Valores y, por otra parte, la tasa de instrumentos de inversión sin riesgo, representada por la de Cetes a 28 días.

Ante los más de cien indicadores que existen en el análisis técnico, después de un análisis exploratorio de todos ellos, decidí evaluar la utilidad como generadores de señales de compra y venta de acciones de sólo 5 indicadores, tres de corto plazo y dos de largo plazo, que se encuentran entre los más comúnmente utilizados en el medio bursátil. Los tres de la primera categoría fueron el oscilador estocástico $\% \mathrm{~K}$ de Lane, el índice de fuerza relativa y la tasa de cambio. En la categoría de indicadores de tendencia (mediano o largo plazo) se incluyó la familia de los osciladores de precios y que son, básicamente, sistemas de cruce de promedios

${ }^{2}$ La palabra momentum significa en inglés, de acuerdo con el Webster's New World Dictionary of American English (Third College Edition, Prentice Hall, EUA, 1994), entre otras cosas: "1. The impetus of a moving object, 2. Strength or force that keeps growing (a campaign that gained momentum), 3. Physics, Mech., the product of the mass of a particle, body, etc., and its velocity". (1. El ímpetu de un objeto en movimiento, 2. Potencia o fuerza que sigue creciendo (una campaña que gana momentum), 3. Física, mecánica, el producto de la masa de una partícula, cuerpo, etc., por su velocidad). Se anotan aquí estos significados porque en español no existe la palabra momentum y la definición de "momento" de la edición de 1997 del diccionario El Pequeño Larousse Ilustrado en Color no contempla estas interpretaciones de "impulso" o "potencia", que son las que se usan en el vocablo inglés.

${ }^{3}$ Díaz Mata, Alfredo. Un sistema para análisis técnico de acciones en la Bolsa Mexicana de Valores. Tesis para obtener el grado de Maestro en Administración en la Facultad de Contaduría y Administración de la Universidad Nacional Autónoma de México, 2002. 
móviles, incluyendo el MACD (Convergencia/Divergencia de promedios móviles) y los cinco elementos del sistema de movimiento direccional creado por Welles J. Wilder.

Las evaluaciones se realizaron simulando con el paquete Metastock las mejores condiciones para comprar y vender acciones de alta bursatilidad de la Bolsa Mexicana de Valores para tres periodos, de 3, 6 y 12 meses que terminaron, todos, el 16 de octubre de 2001. Estas simulaciones se llevaron a cabo evaluando los rendimientos que se pueden obtener tomando decisiones de acuerdo con las señales que generan los indicadores utilizando los valores óptimos de los parámetros que los definen.

Los rendimientos óptimos que se obtuvieron en ese proceso de simulación son los que permiten evaluar la conveniencia o inconveniencia de utilizar los diferentes indicadores como generadores de señales de compra y venta, al compararlos con los rendimientos que se hubieran obtenido al invertir en un instrumento de renta fija como los Cetes a 28 días o el Índice de Precios y Cotizaciones de la Bolsa Mexicana de Valores como punto de referencia del promedio del rendimiento del mercado.

En los tres periodos considerados, el IPC mostró un comportamiento a la baja, es decir, el precio de cierre del 16 de octubre de 2001 fue inferior al precio de cierre de las otras tres fechas anteriores que delimitan los periodos de 3, 6 y 12 meses. Específicamente, los precios de cierre de estas fechas, 16 de octubre de 2000, 16 de abril de 2001 y 16 de julio de este mismo año fueron, todos, superiores a los del 16 de octubre de 2001, por lo que los rendimientos promedio de la bolsa, de acuerdo con su IPC, fueron negativos en los tres casos. Por esta razón, ya no se hicieron comparaciones contra este punto de referencia y sólo se hicieron las comparaciones siguientes contra el rendimiento en la inversión sin riesgo que representan los Cetes a 28 días.

El uso del oscilador estocástico \% K de Lane como base para avanzar en la construcción del sistema de toma de decisiones de inversión en acciones bursátiles que se propone en el presente trabajo es resultado de la conclusión más destacada de aquel estudio: las señales para compra y venta generadas por el oscilador estocástico $\% \mathrm{~K}$ fueron consistentemente mejores que las provenientes de todos los demás indicadores analizados: índice de fuerza relativa, tasa de cambio, los osciladores de promedios móviles utilizados (cruces de promedios móviles, incluyendo el MACD 
Una propuesta para un sistema de análisis técnico para compra y venta de acciones en la Bolsa Mexicana de Valores

con optimización), y los procedimientos sugeridos por el Sistema de Movimiento Direccional, tanto los que sugiere el propio autor del Sistema, Welles J. Wilder, cruce de indicadores de movimiento direccional y el sistema mismo, como este sistema de movimiento direccional modificado, según lo propone el manual del usuario del paquete Metastock. Los rendimientos que se generaron al seguir las señales del \% K fueron también mucho mejores, en términos generales, que la inversión sin riesgo (Cetes a 28 días) y, por supuesto, como se anota arriba, mejor que el rendimiento promedio de la bolsa, medido por el IPC.

Ahora, con respecto al punto de que "las simulaciones se llevan a cabo utilizando, por lo general, periodos de tres meses", esto se debe a que la segunda conclusión importante de la tesis de maestría es que cuanto menor es el plazo mejor es el rendimiento. Y este comportamiento se observó en los tres plazos de 3, 6 y 12 meses en los que se realizaron simulaciones. Esto, además, concuerda con la orientación de corto plazo que tiene el análisis técnico en general.

En la figura 1 se presenta una gráfica con los precios de Bimbo A, de finales de 2003 al 26 de enero de 2004 y se incluye la gráfica del oscilador estocástico dibujada con los parámetros óptimos según se desprenden del proceso de simulación y optimización que se emplea en el sistema propuesto. Este proceso consiste en hacer corridas en la computadora incluyendo rangos de variación para los parámetros variables del oscilador (el número de periodos, n; el número de periodos de frenado, f; la cota inferior, CI; y la cota superior, CS), lo cual arroja decenas de miles de simulaciones, cada una de las cuales arroja información con respecto a los rendimientos que se hubieran obtenido al haber tomado decisiones de compra y venta con base en el comportamiento del oscilador en cada una de esas decenas de miles de configuraciones posibles de los parámetros y se escoge como configuración óptima la que arroja el mayor rendimiento efectivo. El mecanismo para tomar esas decisiones consiste en comprar cuando el oscilador estocástico (la línea punteada) cruza hacia arriba a la cota inferior (la línea horizontal de la parte de abajo) y en vender cuando cruza hacia abajo la cota superior (la línea horizontal de arriba).

Así, en esta figura 1, se habría generado una señal de compra el 24 de octubre de 2003 y se puede ver que los precios de Bimbo A empezaron a subir hacia esa fecha. También se generó una señal de venta el 13 de noviembre, lo cual marcó el comienzo de una racha a la baja. Finalmente, apareció una señal de compra el 25 de noviembre, la cual no ha sido contrarrestada por una de venta hasta la fecha final de la gráfica, el 26 de enero de 2004. 
Otro detalle del sistema que vale la pena anotar aquí es que las simulaciones con este indicador se realizan ya no con el paquete Metastock como inicialmente se hacía, sino con el paquete de Visual Basic, con lo cual se tiene un gran ahorro en el tiempo invertido en esta labor.

Figura 1. Precios de Bimbo A y oscilador estocástico \% K de Lane

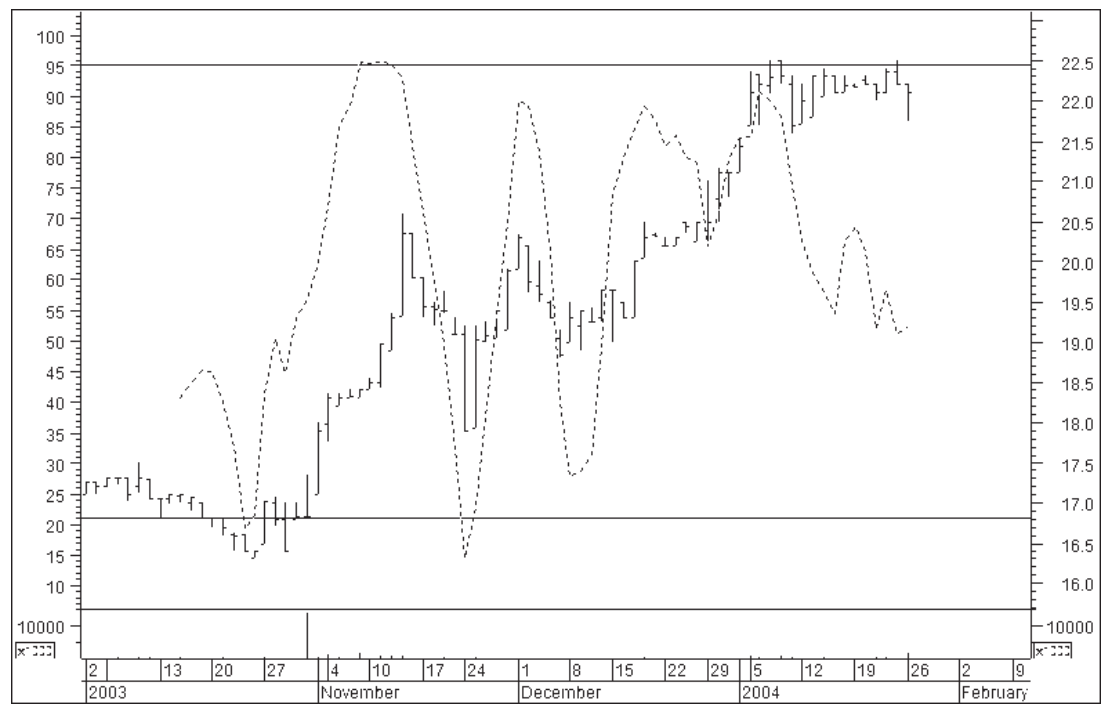

\section{El uso de los indicadores direccionales ID+ e ID-}

Utilicé antes estos indicadores, que forman parte del Sistema de Movimiento Direccional que propone Welles J. Wilder, aunque en forma diferente a como lo propongo ahora. En la tesis utilicé el mecanismo del cruce de esos dos indicadores, tal como sugiere su autor, aunque en forma muy limitada a casos específicos y poco comunes y obtuve resultados aceptables. Sin embargo, con una mayor práctica en su uso encontré que, en muchos casos, son capaces de dar indicación de un cambio en la tendencia casi desde el momento (día) mismo en que comienza y es por ello que los incluyo ahora como parte del sistema que propongo.

El sistema de movimiento direccional fue propuesto por Welles J., Wilder en su libro New Concepts in Technical Trading Systems, publicado por Trend Research, 
Una propuesta para un sistema de análisis técnico para compra y venta de acciones en la Bolsa Mexicana de Valores

en Estados Unidos, en 1978 y es muy utilizado. La idea básica del sistema es resaltar la importancia que tiene para el análisis técnico y, en consecuencia, para la toma de decisiones de inversión, que el mercado o una acción determinada se encuentre en una etapa con tendencia definida (a la alza o la baja) o que se encuentre en un "periodo de congestión", en el que no muestra tendencia definida sino que, más bien, mantiene un movimiento horizontal con alzas y bajas dentro de una especie de banda horizontal.

Una manera de resumir el cálculo del movimiento direccional sería decir que es la porción más grande del rango del día que está fuera del rango del día anterior, donde el rango es la diferencia entre el máximo y el mínimo de la sesión: Se ilustra en la figura 2 en donde se muestran todos los casos posibles de movimiento direccional que se pueden dar en dos sesiones consecutivas.

El caso I de la figura 2 es el ejemplo más sencillo y evidente de movimiento direccional al alza. La magnitud del movimiento direccional positivo (MD+) es la diferencia C-A, tal como se muestra. En el caso II se ilustra un movimiento direccional negativo (MD-) que se mide como la diferencia D-B.

En las ilustraciones III y IV se tienen dos casos de "días exteriores" en los que el rango de los valores máximo y mínimo de la última sesión rebasa en ambos extremos a los valores máximo y mínimo de la sesión anterior. En estos casos, el movimiento direccional se identifica como la diferencia que es mayor entre las diferencias entre los dos máximos y los dos mínimos: en el caso III se tiene un movimiento direccional positivo, MD+ porque la diferencia entre los dos máximos es mayor que la diferencia entre los dos mínimos y, por el contrario, en el caso IV, se tiene un MD- porque es mayor la diferencia entre los mínimos que entre los máximos.

Los casos V y VI son ejemplos de "días internos" en los que el máximo del día es menor o igual que el máximo de la sesión anterior y el mínimo del día es mayor o igual que el mínimo del día anterior. En estas circunstancias resulta claro que no hay tendencia y, por lo tanto, el valor del movimiento direccional es cero.

Finalmente, las ilustraciones VII y VIII muestran "días límite” o "días brecha” y se dan cuando el mínimo del día es igual o mayor que el máximo de la sesión anterior (día límite o día brecha, respectivamente, con MD+) y, por otro lado, cuando el máximo de la sesión es menor o igual que el mínimo de la sesión anterior (día límite o brecha, respectivamente, con MD-). 
Y, entonces, tal como se anota al principio de esta sección, una manera de resumir el cálculo del movimiento direccional consiste en decir que es la porción más grande del rango del día que está fuera del rango del día anterior.

A partir de estas ideas y junto con el concepto del rango verdadero, Wilder construye varios conceptos: el indicador direccional (ID), el índice de movimiento direccional (DX), el índice de movimiento direccional promedio (ADX) y el índice de selección de básicos, uno sobre otro. 
Una propuesta para un sistema de análisis técnico para compra y venta de acciones en la Bolsa Mexicana de Valores

Figura 2. Los casos posibles de movimiento direccional de precios en dos sesiones consecutivas

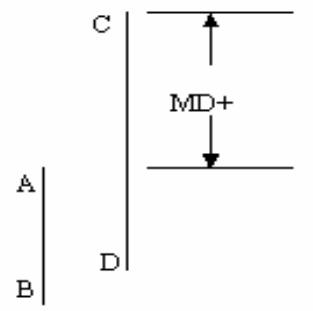

Fig. I

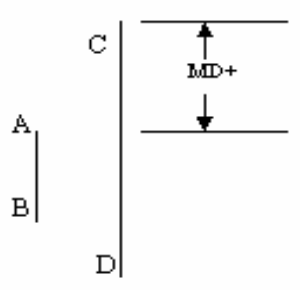

Fig. III

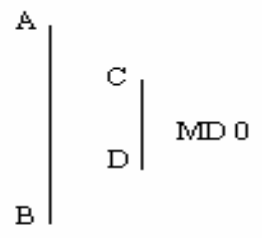

Fig. V

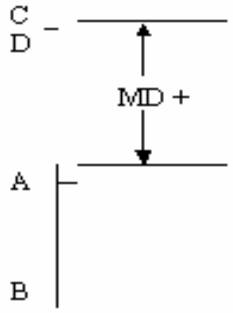

Fig. VII

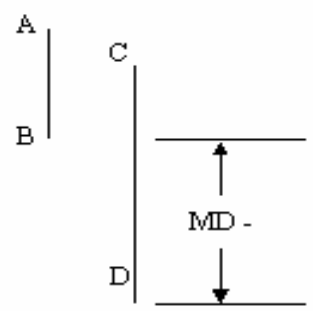

Fig. II

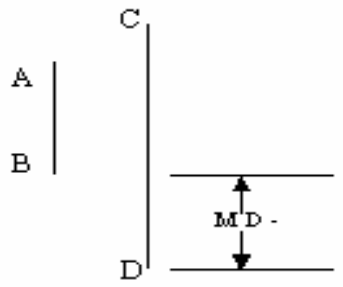

Fig. IV

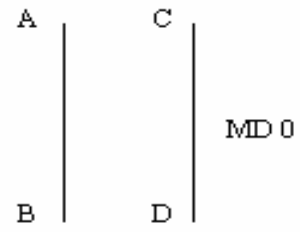

Fig. VI

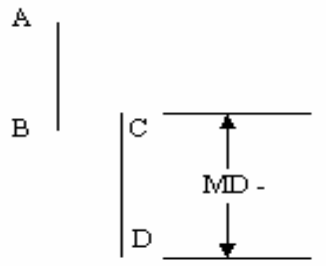

Fig. VIII 
El rango verdadero (RV) se define como el mayor valor que resulte de entre:

1. La diferencia entre el máximo y el mínimo del día

2. La diferencia entre el máximo del día y el cierre del día anterior, y

3. La diferencia entre el mínimo del día y el cierre del día anterior.

O, en símbolos:

1. $\operatorname{Max}_{i}-\operatorname{Min}_{i}$

2. $\left|\operatorname{Max}_{i}-C_{i-1}\right|$

3. $\left|\operatorname{Min}_{i}-C_{i-1}\right|$

Nótese en estos símbolos que, en los casos 2 y 3, se incluyó la simbología del valor absoluto porque interesa sólo la magnitud de la diferencia y no el signo.

\section{El indicador direccional}

Después de haber definido el movimiento direccional positivo (MD+), el movimiento direccional negativo (MD-), y el rango verdadero (RV), Wilder construye con ellos el primer indicador direccional, el más sencillo, que se llama, precisamente, "indicador direccional", que se puede representar como "ID":

$I D+=\frac{M D+}{R V}$

$I D-=\frac{M D-}{R V}$

Nótese en las expresiones anteriores los signos + y - no son operadores aritméticos, sino que sólo sirven para determinar el sentido del movimiento o del índice direccionales.

Estos valores de los índices direccionales positivo y negativo se suman en forma independiente (los positivos por un lado y los negativos por el otro) para obtener totales acumulados que permiten medir qué tanto del movimiento direccional va en 
Una propuesta para un sistema de análisis técnico para compra y venta de acciones en la Bolsa Mexicana de Valores

cada sentido. Desde ahora se puede apreciar que una suma alta de varios ID+ necesariamente implica una suma baja de ID- y se concluiría que el mercado o el título base de los cálculos tienen marcada tendencia alcista. Y, por supuesto, aplica lo contrario cuando la suma de los ID- es alta.

El autor del sistema sugiere utilizar 14 periodos para calcular la suma porque "es un periodo promedio de ciclo medio". En el procedimiento que sugiere Wilder, el primer paso consistiría en construir los dos indicadores direccionales sumando todos los MD+ y todos los MD- y, después, dividiendo cada uno de ellos entre la suma de los 14 rangos verdaderos correspondientes. Y propone, además, que el cálculo de los ID+ e ID- subsecuentes no se lleve a cabo repitiendo el procedimiento anterior, sino utilizando un método de suavización que consiste, básicamente, en restar una porción al ID del periodo anterior y sumarle o restarle, según sea el caso, el movimiento direccional del día. Haciendo esto se tendría, en símbolos:

$I D+{ }_{14}=\frac{M D+_{14}}{R V_{14}}=\frac{\sum_{i=1}^{14} M D+_{i}}{\sum_{i=1}^{14} R V_{i}}, \mathrm{y}$

fórmula 1

$I D-_{14}=\frac{M D-_{14}}{R V_{14}}=\frac{\sum_{i=1}^{14} M D-_{i}}{R V_{i}}$

fórmula 2

Y, entonces, después de haber calculado los primeros índices direccionales de 14 días se procede de la siguiente manera:

$M D 14+_{i}=M D 14+_{i-1}-\frac{M D 14+{ }_{i-1}}{14}+M D_{i} \quad$ fórmula 3

$M D 14-_{i}=M D 14-_{i-1}-\frac{M D 14-{ }_{i-1}}{14}+M D_{i} \quad$ fórmula 4 
Lo que las fórmulas anteriores resumen es el procedimiento para calcular cualquier valor del movimiento direccional, a partir del segundo y que, como puede apreciarse, es igual para el MD+ y el MD-.

El valor del nuevo movimiento direccional es igual al movimiento direccional anterior, menos este mismo dividido entre 14 (su promedio de 14 periodos) y más el movimiento direccional del día. Se aplicaría la primera fórmula si el movimiento direccional es positivo y la segunda si es negativo. En las fórmulas anteriores se usó el valor de 14 que es el que sugiere Wilder.

Los procedimientos anteriores, que resumen la forma de calcular los índices direccionales, tienen dos ventajas. En primer lugar, permiten mantener cierta "memoria" de todos los valores anteriores porque usan el valor anterior (del RV, el MD y el ID) para calcular los siguientes. Una segunda ventaja que menciona Wilder (aunque en realidad ya no tiene mayor importancia en la actualidad) es que se ahorran cálculos y no es necesario repetir los cálculos para los n días o los 14 sugeridos por Wilder.

Y en este punto, según una traducción del texto de Wilder:

Aquí está el verdadero avance -EL VERDADERO MOVIMIENTO DIRECCIONAL es la DIFERENCIA ENTRE ID $+_{14}$ e ID $-_{14}$. Éste es el concepto importante. Conforme más direccional sea el movimiento de un artículo básico (commodity) o una acción, mayor es la diferencia entre ID $+_{14}$ e ID $-{ }_{14}$. En los días en los que se tiene un movimiento direccional positivo, se suma a ID $+_{14} y$, al mismo tiempo, se resta de $I D-{ }_{14}$. Si el sentido del movimiento fuera ascendente durante 14 o más días consecutivos, ID $+_{14}$ tendría un valor alto e ID $-{ }_{14}$ tendería a cero. Por ello, la diferencia entre ambos sería muy grande. ${ }^{4}$

Hasta aquí la traducción del texto de Wilder. El autor abunda diciendo que, si los movimientos consecutivos fueran a la baja se estaría sumando a $I D-{ }_{14}$ y restando a $I D+{ }_{14}$, lo cual también produce los resultados que menciona. Además, hace notar que si los precios tuvieran un comportamiento más o menos lateral (sin tendencia), la diferencia entre los dos indicadores sería reducida lo cual señalaría, precisamente, esa falta de tendencia.

${ }^{4}$ Wilder, Welles J., New Concepts in Technical Trading Systems, Trend Research, Estados Unidos, 1978, p. 39. 
Una propuesta para un sistema de análisis técnico para compra y venta de acciones en la Bolsa Mexicana de Valores

La modificación en la forma de utilizar estos indicadores direccionales, y que considero que arroja mejores resultados, proviene de la observación de que,

a) Cuando los precios han estado bajando considerablemente se espera que de un momento a otro comiencen a subir. Esta expectativa de cambio es fácil de justificar si se observa cualquier gráfica de precios de acciones bursátiles. Los precios suben y bajan constantemente y la duración de estas rachas a la alza y a la baja varía enormemente. ${ }^{5}$

b) Cuando los precios han estado bajando considerablemente, los valores de estos dos indicadores se encuentran en los extremos. En la figura 3 se muestra la gráfica de precios de GFNorte O, de fines de 2003 hasta el 26 de enero de 2004 y se incluyen las líneas correspondientes a DI+ (la línea continua) y DI- (la línea punteada).

Figura 3. Precios de GFNorte $\mathrm{O}$ y sus indicadores direccionales

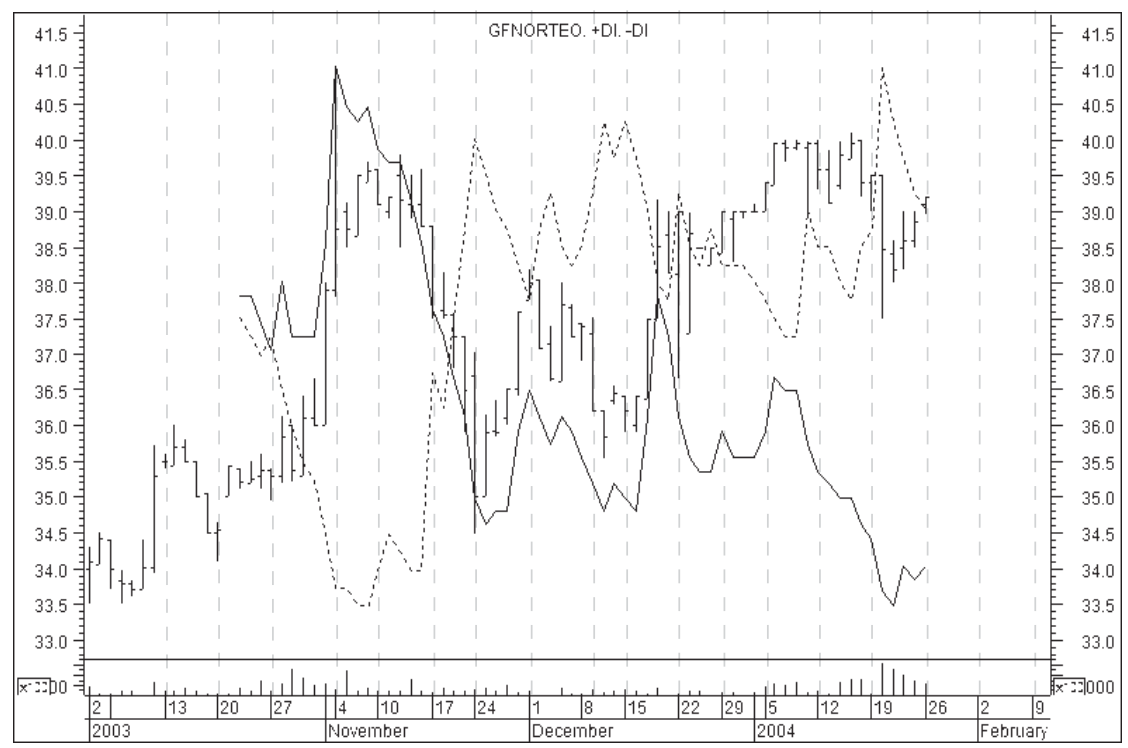

${ }^{5}$ Esto tiene que ver, entre otros temas, con la clasificación de las tendencias que se hace en la Teoría Dow, de acuerdo con su duración: primaria (más de 3 meses), secundaria (3 semanas a 3 meses) y menor (de tres semanas). Se relaciona también con la muy popular teoría de las olas de Elliott y es un tema que se aborda prácticamente en cualquier texto sobre análisis técnico. 
En esta gráfica se observa, por un lado, que la racha a la baja que comenzó a principios de noviembre estuvo marcada por valores extremadamente altos del ID- y extremadamente bajos del ID+ y que, cuando estos dos indicadores comenzaron a revertir estas posiciones, los precios de la acción comenzaron a bajar. Por otro lado, en la semana que comenzó el 19 de enero de 2004, los dos indicadores se encontraban en posiciones extremas también, pero contrarias a las anteriores y el comienzo de un cambio en su tendencia también marcó el inicio de otra racha ascendente en los precios de las acciones de GFNorte O.

Así, el análisis de muchas gráficas de precios, para muchos periodos de las 29 acciones que he venido siguiendo desde hace año y medio, me permite concluir que estos valores extremos de los indicadores direccionales suelen ser el inicio de un cambio en la tendencia de los precios, especialmente cuando suceden simultáneamente. Esto, por supuesto, se puede observar al día siguiente del "quiebre" y que equivaldría a la señal de compra o de venta. Una muestra de fortaleza adicional en estas señales de los indicadores direccionales se da cuando los quiebres se producen después de una tendencia marcada y prolongada. En otras palabras, cuanto más larga y prolongada es la tendencia que precede a los quiebres, más fuerte es la señal que se genera.

\section{Las acciones que generan señales}

En primer lugar, se determina si hay alguna acción en la que se presenten simultáneamente señales coincidentes (de compra o de venta) con los dos indicadores principales del sistema, el oscilador \% $\mathrm{K}$ y los indicadores direccionales. Este mecanismo de buscar coincidencias entre las señales es otro elemento importante de la propuesta que aquí se plantea y coincide con otro principio ampliamente aceptado en el análisis técnico y que afirma que una coincidencia entre distintos indicadores fortalece las señales, lo cual tiene, además, un sentido lógico natural. Recapitulando, el oscilador estocástico \% K mide, básicamente, qué tanto varía el precio de cierre actual con respecto a los máximos y mínimos de $\mathrm{n}$ periodos previos, en tanto que los indicadores direccionales miden qué tanto han estado variando los precios de un día a otro y si lo han estado haciendo al alza o a la baja, y considero que este último planteamiento permite comprender de mejor manera cómo es que se complementa la información de ambos indicadores y cómo es que su uso conjunto permite mejorar la toma de decisiones. 
Una propuesta para un sistema de análisis técnico para compra y venta de acciones en la Bolsa Mexicana de Valores

Así, la primera indicación, y la más importante, de que se podría tener una señal de compra o de venta se da cuando coinciden las señales de estos dos indicadores y, dependiendo de esto, se amplía el análisis para determinar si existen indicaciones adicionales que fortalezcan esa primera e importante señal (por supuesto, las señales de venta se toman en cuenta sólo cuando se poseen acciones de las que generan la señal).

Adicionalmente se buscan otras dos señales que pueden fortalecer las señales y que son las brechas y los días clave de reversión, que se explican en los párrafos siguientes.

\section{Brechas}

Las brechas son señales de fortaleza en una tendencia alcista y de debilidad en una a la baja y se trata de pares de días consecutivos en los que la barra de precios del primer día da un salto (brecha) con respecto a la barra de precios del día siguiente. En otras palabras, no hay traslape entre los precios máximos o los precios mínimos de días consecutivos.

Hay tres tipos de brechas:

1. Brecha de rompimiento (breakaway) Se da al inicio de un alza y usualmente con volumen alto.

2. Brecha de fuga (runaway) o de medición (measuring) Se da en medio de una tendencia e indica que los precios se están moviendo fácilmente con poco volumen.

3. Brecha de agotamiento (exhaustion). Normalmente se da al final de una racha alcista.

En la figura 4 se muestran el caso de una brecha de fuga que se dio entre el 2 y el 5 de enero de 2004, en los precios de AMX L. 
Figura 4. Brechas en los precios deAMX L

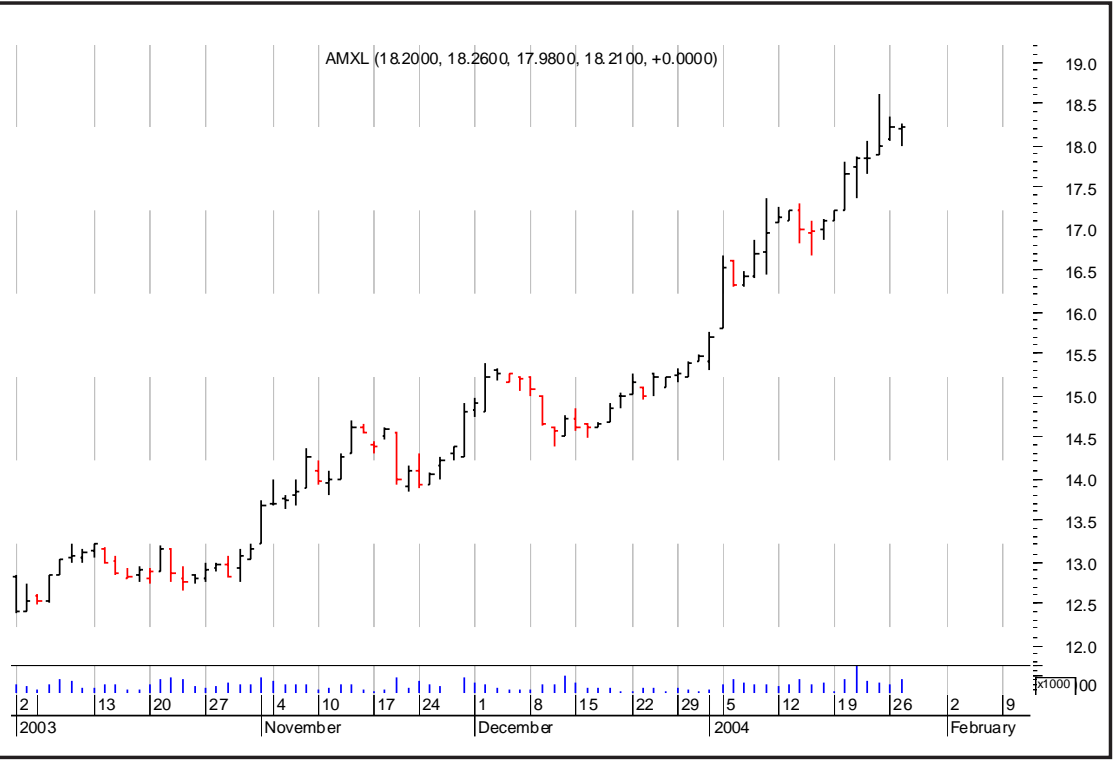

Figura 5. Datos deAra* con dos días cumbre de reversión

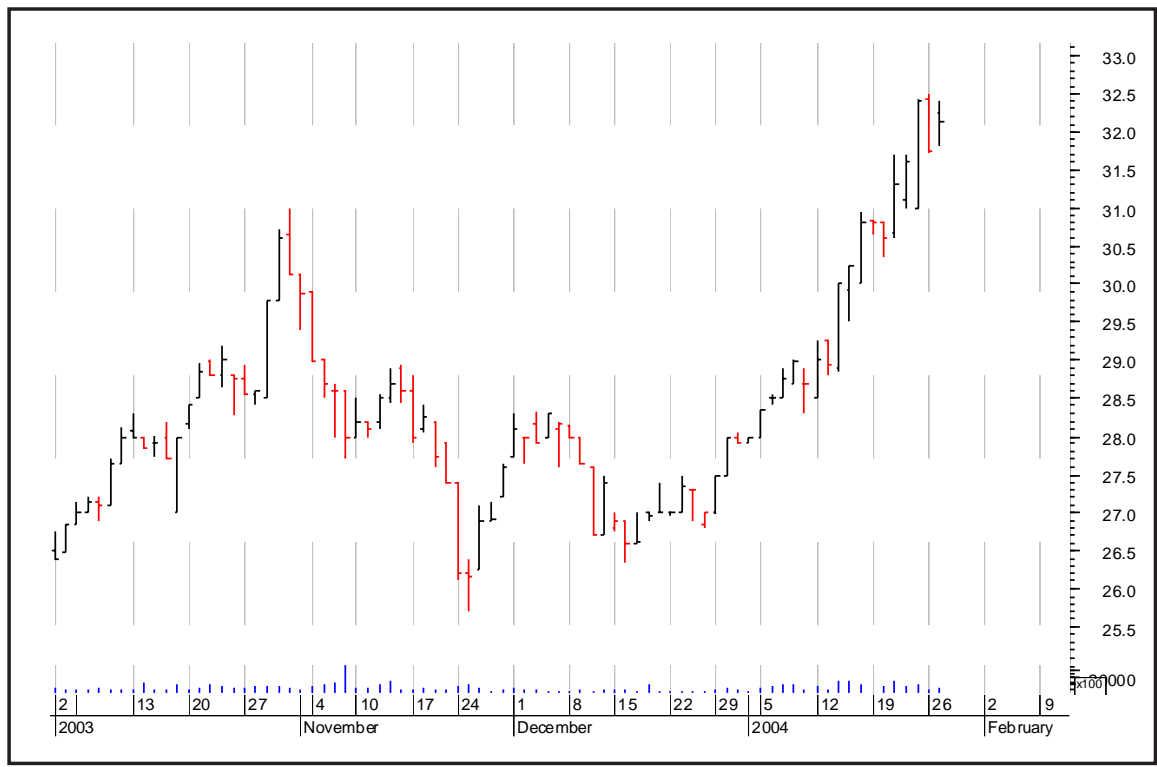


Una propuesta para un sistema de análisis técnico para compra y venta de acciones en la Bolsa Mexicana de Valores

\section{Días clave de reversión}

Éste es otro mecanismo que he venido incluyendo en los análisis porque parece ofrecer información adicional y fácil de visualizar sobre el posible comportamiento futuro de los precios. Un día cumbre de reversión es cuando en una tendencia alcista se llega a un nuevo máximo, seguido de un cierre menor al del día anterior. Por el contrario, un día valle de reversión sucede cuando en una tendencia a la baja se llega a un nuevo mínimo, seguido de un cierre superior al del día anterior. Para resumir ambos conceptos en uno, se habla de "días clave de reversión". En la figura 5 se muestran los precios de Ara*, que incluyen dos días cumbre de reversión, uno el 31 de octubre de 2003 y el otro el 26 de enero de 2004. Nótese cómo ambos días clave de reversión marcan el inicio de una racha descendente.

La determinación de estas brechas y días clave de reversión se hace automáticamente con el programa en Visual Basic.

\section{Evaluación de la tendencia del mercado, a través del IPC y del DJIA}

Este análisis se lleva a cabo utilizando todos los mecanismos mencionados, oscilador estocástico \% $\mathrm{K}$, indicadores direccionales, días de reversión y brechas.

\section{EL DESEMPEÑO DEL SISTEMA}

Se ha venido utilizando el sistema descrito para comprar y vender acciones en la Bolsa Mexicana de Valores desde noviembre de 2002, en una cuenta abierta en la casa de bolsa Accival, a través de su sitio en Internet, Accitrade, mediante el cual se pueden llevar a cabo las operaciones desde una computadora. En el anexo 1 se presenta el historial de las compras y las ventas realizadas, incluyendo la utilidad o la pérdida de cada transacción, hasta el 27 de enero de $2004^{6}$.

En los últimos renglones de esa tabla se ve que el resultado neto de las compras y las ventas fue de $\$ 53,719.24$ que, para una inversión promedio de $\$ 200,000$ en ese periodo, representa una cantidad considerable y un rendimiento aproximado del $25 \%$, lo cual es un resultado muy alentador porque supera notablemente al

\footnotetext{
${ }^{6}$ La veracidad de estas operaciones queda verificada por estados de cuenta expedidos por la casa de bolsa Accival del Grupo Financiero Banamex, a través de su mecanismo AcciTrade de operaciones por Internet.
} 
rendimiento que se obtiene actualmente en inversiones de renta fija, como los Cetes a 28 días, que ofrecen $4.69 \%$ anual, al jueves 29 de enero de 2004. Sin embargo, por otro lado es importante tener presente que el rendimiento del Índice de Precios y Cotizaciones de la BMV tuvo un incremento del $55.9 \%$ en ese mismo periodo de 14 meses, al pasar de 6,126.24 a 9,551.13 puntos.

Como un punto de comparación adicional ${ }^{7}$, en la tabla siguiente se muestran los fondos de inversión en renta variable (Sociedades de Inversión Comunes) que ofrecieron rendimientos superiores al marcado por el IPC de la bolsa. La información contenida en esta tabla, que muestra rendimientos de hasta casi el $100 \%$ (GBMCRE DE GBM) para el mismo periodo considerado, evidencia la necesidad de considerar estos rendimientos como el parámetro más desafiante para evaluar el rendimiento del sistema que se está construyendo y para lo cual es necesario, además, tomar en cuenta aspectos adicionales.

Lo primero que es necesario tomar en consideración es que los resultados de estos fondos provienen de empresas que cuentan con recursos e infraestructura muy importante en términos tanto de especialistas bursátiles como de instalaciones y acceso a la información relevante, en tanto que el sistema que aquí se propone está implementado por un inversionista aislado que, además, dispone de poco tiempo para seguir el comportamiento de los mercados (es por esto, entre otras razones, por lo que se propone no utilizar el análisis gráfico).

${ }^{7}$ Sugerido por el maestro Julio Castellanos Elías en el Seminario de Investigadores de la División de Investigación de la FCA, en donde se evaluó este trabajo como paso previo a su publicación. 
Una propuesta para un sistema de análisis técnico para compra y venta de acciones en la Bolsa Mexicana de Valores

\begin{tabular}{|c|c|c|c|c|}
\hline FONDO & OPERADORA & $27 / 01 / 2004$ & $27 / 11 / 2002$ & \begin{tabular}{|l|} 
RENDIMIENTO \\
\end{tabular} \\
\hline GBMCRE & GBM & 1.269687 & 0.651713 & 94.82 \\
\hline ACCIAR & BANAMEX & 66.99748 & 37.758709 & 77.44 \\
\hline STDER-D & SANTANDER & 15.588077 & 9.10923 & 71.12 \\
\hline ING-REN & BARING & 1.690937 & 0.998143 & 69.41 \\
\hline STDER-A & SANTANDER & 2.829979 & 1.688486 & 67.60 \\
\hline ING-PAT & BARING & 2.571365 & 1.539222 & 67.06 \\
\hline LIDER-A & SANTANDER & 9.418037 & 5.640642 & 66.97 \\
\hline VECTCR & S.I.GENERA & 84.4467 & 51.33566 & 64.50 \\
\hline STDER-I & SANTANDER & 8.922125 & 5.443428 & 63.91 \\
\hline ISOLIDO & INTERACCS & 156.417366 & 95.536011 & 63.73 \\
\hline FIRME & SANTANDER & 4.802843 & 2.945551 & 63.05 \\
\hline ACTIPAT & FINACORP & 2.726571 & 1.674952 & 62.79 \\
\hline BINDEX & BURSAMEX & 50.716234 & 31.18089 & 62.65 \\
\hline ING-IPC & BARING & 1.632863 & 1.004728 & 62.52 \\
\hline GBMIPC & GBM & 0.451264 & 0.28015 & 61.08 \\
\hline GBMAAA & GBM & 0.977369 & 0.607613 & 60.85 \\
\hline BMERPAT & BANCOMER & 22.094061 & 13.7901 & 60.22 \\
\hline VALMX20 & VALMEX & 2.198981 & 1.372742 & 60.19 \\
\hline SCOTIA7 & INVERLAT & 7.446247 & 4.649301 & 60.16 \\
\hline ACTIVAR & ACTINVER & 18.871552 & 11.794849 & 60.00 \\
\hline NORTEIN & BANORTE & 7.285504 & 4.576691 & 59.19 \\
\hline VECTIND & S.I.GENERA & 25.75354 & 16.23494 & 58.63 \\
\hline BMERIND & BANCOMER & 22.710854 & 14.342206 & 58.35 \\
\hline ZCAP4 & INVEX & 1.52625 & 0.974978 & 56.54 \\
\hline BOSTONB & BANKBOSTON & 1.411475 & 0.906121 & 55.77 \\
\hline VAINDEX & VANGUARDIA & 1.560775 & 1.008766 & 54.72 \\
\hline ZCAP1 & INVEX & 81.753182 & 53.234309 & 53.57 \\
\hline VECTPA & S.I.GENERA & 127.49465 & 83.59126 & 52.52 \\
\hline BMERTOP & BANCOMER & 7.192294 & 4.715667 & 52.52 \\
\hline NAFINDX & NAFINSA & 13.026076 & 8.54347 & 52.47 \\
\hline AWLLOYD & LLOYD & 4.518574 & 2.968431 & 52.22 \\
\hline IXECON & IXE & 0.053808 & 0.035372 & 52.12 \\
\hline VALUEV6 & VALUE & 55.70746 & 36.64423 & 52.02 \\
\hline SCOTIA6 & INVERLAT & 5.098081 & 3.364335 & 51.53 \\
\hline IXECAP & IXE & 2.699433 & 1.803204 & 49.70 \\
\hline ZCAP2 & INVEX & 44.052789 & 29.438988 & 49.64 \\
\hline NORTERV & BANORTE & 0.815784 & 0.547848 & 48.91 \\
\hline VALMX24 & VALMEX & 1.051794 & 0.713728 & 47.37 \\
\hline BITALV4 & BITAL & 13.722231 & 9.383762 & 46.23 \\
\hline F-INDIC & APOLO & 41.307701 & 28.540157 & 44.74 \\
\hline ACCIPAT & BANAMEX & 182.91722 & 126.480844 & 44.62 \\
\hline NORTEDE & BANORTE & 0.754522 & 0.523686 & 44.08 \\
\hline CITIPAT & BANACCI & 1.430947 & 0.99516 & 43.79 \\
\hline
\end{tabular}




\begin{tabular}{|ll|r|r|r|}
\hline GBMV2 & GBM & 3.90247 & 2.793477 & 39.70 \\
\hline VACRECI & VANGUARDIA & 1.524549 & 1.091362 & 39.69 \\
\hline STDER-M & SANTANDER & 0.568046 & 0.407529 & 39.39 \\
\hline ACTIDOW & FINACORP & 1.593463 & 1.158413 & 37.56 \\
\hline FONBNM & BANACCI & 30.687919 & 22.35093 & 37.30 \\
\hline BMIL & BIURSAMEX & 28.336036 & 20.81069 & 36.16 \\
INCOMUN & INTERESA & 7.171782 & 5.272309 & 36.03 \\
\hline BOSTONP & BANKBOSTON & 1.212587 & 0.893079 & 35.78 \\
ACCIVAL & BANAMEX & 118.743753 & 88.388695 & 34.34 \\
\hline ICAPTAL & INTERACCS & 4.165716 & 3.103302 & 34.23 \\
BMERBAL & BANCOMER & 14.648762 & 11.145481 & 31.43 \\
\hline MVBOLSA & CBMULTI & 0.819676 & 0.62429 & 31.30 \\
\hline MONEXRV & O.MONEX & 0.051795 & 0.03998 & 29.55 \\
\hline VALUEV5 & VALUE & 5.67027 & 4.41486 & 28.44 \\
\hline BITALV3 & BITAL & 10.007198 & 7.835697 & 27.71 \\
\hline GBMV1 & GBM & 7.134094 & 5.764292 & 23.76 \\
\hline GBMBAL & GBM & 1.286913 & 1.063634 & 20.99 \\
BMERCRE & BANCOMER & 35.11672 & 29.537275 & 18.89 \\
\hline VALMX22 & VALMEX & 6.816462 & 5.793853 & 17.65 \\
F-BOLSA & APOLO & 1.99301 & 1.705621 & 16.85 \\
\hline BITALV2 & BITAL & 3.064409 & 2.681217 & 14.29 \\
\hline CITIFLE & BANACCI & 0.974595 & 0.861902 & 13.07 \\
\hline ICAMIX & IVERCAP & 0.765453 & 0.690311 & 10.89 \\
\hline ZEVERST & INVEX & 0.970076 & 0.883661 & 9.78 \\
\hline PROMEX5 & BANCOMER & 104.734357 & 96.456245 & 8.58 \\
\hline VALOR4C & VALORUM & 0.04153 & 0.041028 & 1.22 \\
\hline ARKAPAQ & ARKA & 1.190349 & 55.76876 & -5.12 \\
\hline OFINCRE & OFIN & 0.348654 & 0.369824 & -13.10 \\
\hline APOLO10 & APOLO & 0.92007 & 1.21031 & -23.98 \\
\hline VALMX26 & VALMEX & 5.732069 & 378.827294 & -83.44 \\
VECTPRO & S.I.GENERA & 35.070001 & -83.45 \\
\hline FONIBUR & INBURSA & & & -84.27 \\
ACTICRE & ACTINVER & & & \\
\hline INBURSA & INBURSA & & 191.189119 & \\
\hline
\end{tabular}

Adicionalmente, para obtener los rendimientos observados en los fondos de inversión es necesario también conocer el mercado para poder aprovechar los periodos alcistas y decidir en cuál de ellos invertir porque, tal como puede observarse en la tabla anterior, hubo fondos que no sólo no alcanzaron el rendimiento mostrado por el IPC sino que, incluso, incurrieron en pérdidas en el mismo periodo (-84.27\% en el caso del fondo INBURSA). 
Una propuesta para un sistema de análisis técnico para compra y venta de acciones en la Bolsa Mexicana de Valores

Por otro lado, y al mismo tiempo, hay varias consideraciones que, una vez incorporadas al sistema, deberán permitir mejorar considerablemente su eficiencia y que es lo que se está trabajando en la actualidad:

- El mecanismo para identificar tendencias marcadas que se mencionó antes.

- Mecanismos para detener las pérdidas en los casos en los que una compra de acciones no vaya seguida del movimiento alcista esperado.

- Algún indicador o indicadores que incluyan el volumen en los análisis.

\section{BIBLIOGRAFÍA}

Díaz Mata, Alfredo y Víctor Manuel Aguilera Gómez, Matemáticas Financieras, tercera edición, Libros Mc-Graw Hill, México, 1999.

Invierta en la bolsa. Guía para inversiones seguras y productivas, segunda edición, Grupo Editorial Iberoamérica, México, 1994.

Un sistema computarizado para análisis de acciones bursátiles en México, tesis inédita para obtener el grado de Maestro en Finanzas, Facultad de Contaduría y Administración, Universidad Nacional Autónoma de México, 2002.

"Los osciladores \% $\mathrm{K}$ y \% R del análisis técnico bursátil y una propuesta para mejorar el \%R", Contaduría y Administración, Facultad de Contaduría y Administración, Universidad Nacional Autónoma de México, número 203, octubre-diciembre de 2001.

"Un esquema propuesto para el análisis bursátil de acciones en la Bolsa Mexicana de Valores", Contaduría y Administración, Facultad de Contaduría y Administración, Universidad Nacional Autónoma de México, número 189, abril-junio de 1998.

"El posible inicio de una racha descendente en el mercado accionario bursátil mexicano y la especulación con instrumentos financieros derivados", Contaduría y Administración, Facultad de Contaduría y Administración, Universidad NacionalAutónoma de México, número 187, octubre-diciembre de 1997. 
"Panorama actual del análisis bursátil de acciones", Contaduría y Administración, Facultad de Contaduría y Administración, Universidad Nacional Autónoma de México, número 184, enero-marzo de 1997.

Edwards, Robert y John Magee, Technical Analysis of Stock Trends, octava edición, John Magee, Inc. EUA, 2001. (La primera edición es de 1948).

Metastock, User's Manual, versión 8.0, Equis Internacional, Inc., EUA, 2002.

Murphy, John J., Technical Analysis of the Financial Markets, New York Institute of Finance, edición revisada de Technical Analysis of The Futures Markets (1986), EUA, 1999.

Pring, Martin J., Technical Analysis Explained, cuarta edición, McGraw Hill, EUA, 2002.

Sánchez Cantú, Leopoldo, Claudio Núñez Sánchez de la Barquera y Eduardo Couto Castillo, Invierta con éxito en la bolsa de valores, utilice el análisis técnico, Prentice Hall y NSC Asesores, México, 1999.

Wilder, Welles J., New Concepts in Technical Trading Systems, Trend Research, EUA, 1978. 